\title{
The big lobe of 67P/Churyumov-Gerasimenko comet: morphological and spectrophotometric evidences of layering as from OSIRIS data
}

\author{
Sabrina Ferrari, ${ }^{1 \star}$ L. Penasa,${ }^{1}$ F. La Forgia,${ }^{2}$ M. Massironi, ${ }^{1,3}$ G. Naletto, ${ }^{1,4,5}$ \\ M. Lazzarin, ${ }^{2}$ S. Fornasier, ${ }^{6}$ P. H. Hasselmann, ${ }^{6}$ A. Lucchetti, ${ }^{7}$ M. Pajola, ${ }^{7}$ F. Ferri, ${ }^{1}$ \\ P. Cambianica, ${ }^{1}$ N. Oklay, ${ }^{8}$ C. Tubiana, ${ }^{9}$ H. Sierks, ${ }^{9}$ P. L. Lamy, ${ }^{10}$ R. Rodrigo, ${ }^{11,12}$ \\ D. Koschny, ${ }^{13}$ B. Davidsson, ${ }^{14}$ M. A. Barucci, ${ }^{6}$ J.-L. Bertaux, ${ }^{15}$ I. Bertini, ${ }^{2}$ \\ D. Bodewits, ${ }^{16}$ G. Cremonese, ${ }^{7}$ V. Da Deppo, ${ }^{5}$ S. Debei, ${ }^{17}$ M. De Cecco, ${ }^{18}$ J. Deller, ${ }^{9}$ \\ M. Franceschi, ${ }^{3}$ E. Frattin,,${ }^{2,7}$ M. Fulle, ${ }^{19}$ O. Groussin, ${ }^{20}$ P. J. Gutiérrez, ${ }^{21}$ C. Güttler, ${ }^{9}$ \\ S. F. Hviid, ${ }^{8}$ W.-H. Ip, ${ }^{22,23}$ L. Jorda, ${ }^{20}$ H. U. Keller, ${ }^{24,8}$ J. Knollenberg, ${ }^{8}$ E. Kührt, ${ }^{8}$ \\ M. Küppers, ${ }^{25}$ L. M. Lara, ${ }^{21}$ J. J. López-Moreno, ${ }^{21}$ F. Marzari, ${ }^{4}$ X. Shi, ${ }^{9}$ E. Simioni, ${ }^{7}$ \\ N. Thomas ${ }^{26,27}$ and J.-B. Vincent ${ }^{8}$ \\ Affiliations are listed at the end of the paper
}

Accepted 2018 June 7. Received 2018 May 9; in original form 2018 February 5

\begin{abstract}
Between 2014 and 2016, ESA's Rosetta OSIRIS cameras acquired multiple-filters images of the layered nucleus of comet 67P/Churyumov-Gerasimenko, ranging from ultraviolet to near-infrared wavelengths. No correlation between layers disposition and surface spectral variegation has been observed so far. This paper investigates possible spectral differences among decametre-thickness outcropping layers of the biggest lobe of the comet by means of OSIRIS image dataset. A two-classes maximum likelihood classification was applied on consolidated outcrops and relative deposits identified on post-perihelion multispectral images of the big lobe. We distinguished multispectral data on the basis of the structural elevation of the onion-shell Ellipsoidal Model of 67P. The spatial distribution of the two classes displays a clear dependence on the structural elevation, with the innermost class resulting over 50 per cent brighter than the outermost one. Consolidated cometary materials located at different structural levels are characterized by different brightness and revealed due to the selective removal of large volumes. This variegation can be attributed to a different texture of the outcrop surface and/or to a different content of refractory materials.
\end{abstract}

Key words: methods: data analysis-techniques: image processing-comets: generalcomets: individual: 67P/Churyumov-Gerasimenko.

\section{INTRODUCTION}

Since its rendezvous with comet 67P/Churyumov-Gerasimenko (67P) in 2014, ESA's Rosetta spacecraft provided countless images of the cometary nucleus. During the $2 \mathrm{yr}$ mission, indeed, the Optical, Spectroscopic, and Infrared Remote Imaging System (OSIRIS; Keller et al. 2007) imaged the nucleus surface by means of two different cameras - a Narrow Angle Camera (NAC) and a Wide Angle Camera (WAC), the latter mainly dedicated to gas and dust studies - with filters ranging from ultraviolet to near-infrared wavelengths.

Thanks to such imagery, the identification of morphological properties as the bilobate shape (Sierks et al. 2015) and the series of cliffs and terraces of the nucleus (Massironi et al. 2015; Thomas et al. 2015) has been complemented by detailed studies of the spectrophotometric behaviour of the global surface (Fornasier et al. 2015; Perna et al. 2017). The morphological terraces have been interpreted as the surface expression of extended discontinuities that separate superimposed layers of consolidated material (Massironi et al. 2015; Giacomini et al. 2016; Lee et al. 2016). This implies an 'onionlike' structure of the two lobes (Massironi et al. 2015) suggesting 
that two independent layered objects collided at low velocity merging in a single bulk where the big lobe, called the body, and the small lobe, called the head, are joined through a restricted region, called the neck (Rickman et al. 2015; Jutzi \& Benz 2017). Although partially covered by deposits of unconsolidated materials, layers appear laterally persistent at the regional scale, producing a staircase morphology where shallower layers superimpose layers located at progressively deeper structural levels. At the same time, the global surface of the nucleus was investigated using OSIRIS multispectral data, searching for local and regional variegations of composition and texture. Local spectral inhomogeneities of the 67P nucleus surface have been fully analysed by Oklay et al. (2015), who classified active, inactive, and mixed areas by showing water-ice-rich activity sources, and by Oklay et al. (2016, 2017), who defined properties and survival of the water ice on the surface. Using nucleus images acquired prior to the last perihelion passage of 2015 August, Fornasier et al. (2015) found an overall dark geometric albedo of $6.5 \pm 0.2$ per cent at $649 \mathrm{~nm}$ and confirmed the ground-based observations of a spectrally red featureless cometary nucleus along visible and near-infrared wavelengths (Tubiana et al. 2011). They found heterogeneities both in terms of albedo and colours of the nucleus surface, and they identified three groups of regions, based on the surface spectral slope computed in the 535-882 $\mathrm{nm}$ range, which varies from 10 to 21 per cent at a phase angle of $50^{\circ}$. The relatively bluer (i.e. lower slope value) regions are associated to terrains enriched in water ice content, while redder regions are found mainly in dust covered terrains. Perna et al. (2017) applied a statistical investigation on similar pre-perihelion multispectral data, in order to identify surface properties without a-priori grouping. By means of a multivariate analysis, they identified four groups characterized by different spectral slope ranges, three of which closely match the groups defined by Fornasier et al. (2015). In both cases, groups enhance the relative surface content of volatiles, but no correlation with a layered disposition has been observed.

An additional hint on the pre-perihelion global variegation comes from the Rosetta's Visible and InfraRed Thermal Imaging Spectrometer (VIRTIS; Coradini et al. 2007), which long before the last passage detected dark dry organic-rich refractory materials that uniformly blanketed the nucleus surface (Capaccioni et al. 2015), being possibly the remnant of the strong sublimation at the previous perihelion passages.

The overall pre-perihelion picture of the 67P nucleus thus shows a terraced morphology that, underneath a refractory-enriched skin, displays local and regional variations of the volatiles content (De Sanctis et al. 2015; Fornasier et al. 2016, 2017). These variegations can be in part caused by the diurnal and seasonal variations of the nucleus illumination conditions. The nucleus, indeed, is subject to diurnal cycles of depletion and re-condensation of volatiles in the shallower subsurface (Capaccioni et al. 2015), as observed on different regions of the comet (De Sanctis et al. 2015; Fornasier et al. 2016), as well as more energetic seasonal outgassing activities during every passage close to the Sun (Vincent et al. 2016, and references therein). The high activity close to the perihelion induces a higher erosion rate in the southern hemisphere, and a thinner surface dust coating, partially showing the underlying icerich layer and resulting in an overall less red colour of the nucleus (Fornasier et al. 2016). In particular, during perihelion, the illuminated southern hemisphere is more active than the unlit northern one (Keller et al. 2017), which accumulates airfall that originates from the active south. This implies that nowadays the cometary surface is continuously reworked, and volatiles (De Sanctis et al. 2015), as well as the finest fraction of unconsolidated materials (e.g. Lai et al. 2016; Pajola et al. 2017a), are moved from a site to the other depending on the illumination conditions and nearly independently of any physioregional boundary. In addition, a strong illumination and an effective thermal gradient (Pajola et al. 2017a) induce the exposure of fresh materials that, later, are gradually depleted of their volatiles content (Fornasier et al. 2016; Pajola et al. 2017a).

Besides diurnal and seasonal factors, the spectrophotometric variegation of 67P could depend on its layered structure, which implies the progressive stacking of material during the accretion of the two distinct lobes and, later, the exposition of certain stacks as a result of erosive processes. By measuring the orientation of the terraces, Penasa et al. (2017) produced a simplified three-dimensional ellipsoid-based model (Ellipsoidal Model, EM) that can be used to evaluate the structural level of any point on the cometary surface as a distance from the structural centre of the pertaining lobe. They resolved the overall geometry of the layers, highlighting that different portions of the comet expose different structural depths, thus implying that 67P underwent a non-homogenous process of volume removal. According to the EM, the outermost and innermost layers are revealed in different physiographical regions (El-Maarry et al. 2015 , 2016) of the big lobe, as shown in Fig. 1. The innermost layers correspond to the Imhotep region, whereas the outermost ones are located between Atum and the elongated Ash region, including Apis and part of the Khonsu region. The latter display an abrupt change of albedo with respect to the underlying layers, appearing darker (Ash region, Fig. 1c; e.g. Fornasier et al. 2015) and pervaded by a goosebump texture (Apis region, Fig. 1d; e.g. Davidsson et al. 2016). Fornasier et al. (2015) found that $1 \mathrm{yr}$ before the perihelion passage the outcropping Ash and Apis regions were characterized by a lower albedo and a generally redder slope with respect to the smooth part of Imhotep, which corresponded to a thick cover of fine materials possibly retaining a higher ice content. Apis region and the outcropping portions of Ash and Imhotep, indeed, were included in the high (redder) spectral-slope group, whereas the smooth dusty surfaces of Ash and Imhotep were included in the average spectralslope group. As a whole, Fornasier et al. (2015) concluded that the reflectance was anticorrelated with the spectral slope and the albedo was correlated to the surface texture.

We therefore conducted a spectrophotometric analysis of OSIRIS images based on multispectral data analyses and classifications on those big-lobe outcropping regions, in order to identify possible morphological, textural, and/or compositional characteristics that allow to distinguish regional stacks of layers.

\section{DATASET AND METHODS}

The OSIRIS NAC is equipped with 11 filters ranging from ultraviolet to near-infrared wavelength (250-1000 nm; Keller et al. 2007). In order to cover the widest overview on Imhotep, Ash, Apis, Atum, and Khonsu regions, two NAC image sequences (Fig. 2 and Table 1) were selected, meeting the broadest range of available filters (nine filters for these locations), comparable spatial resolutions (1.33 to $\left.1.71 \mathrm{~m} \mathrm{pixel}^{-1}\right)$, and phase angles $\left(62^{\circ}-65^{\circ}\right)$, and including filters frequently used in previous 67P spectrophotometric studies (Fornasier et al. 2015, 2016, 2017; La Forgia et al. 2015; Oklay et al. 2015, 2016, 2017; Deshapriya et al. 2016, 2018; Feller et al. 2016; Lucchetti et al. 2016, 2017; Pajola et al. 2016, 2017a,b; Hasselmann et al. 2017). Selected images are post-perihelion acquisitions of 2016 January (2.22 au).

On the 67P surface it is possible to distinguish layers, which are formed by consolidated material, and deposits, which are formed by fragments of consolidated materials (Giacomini et al. 2016; Lee 

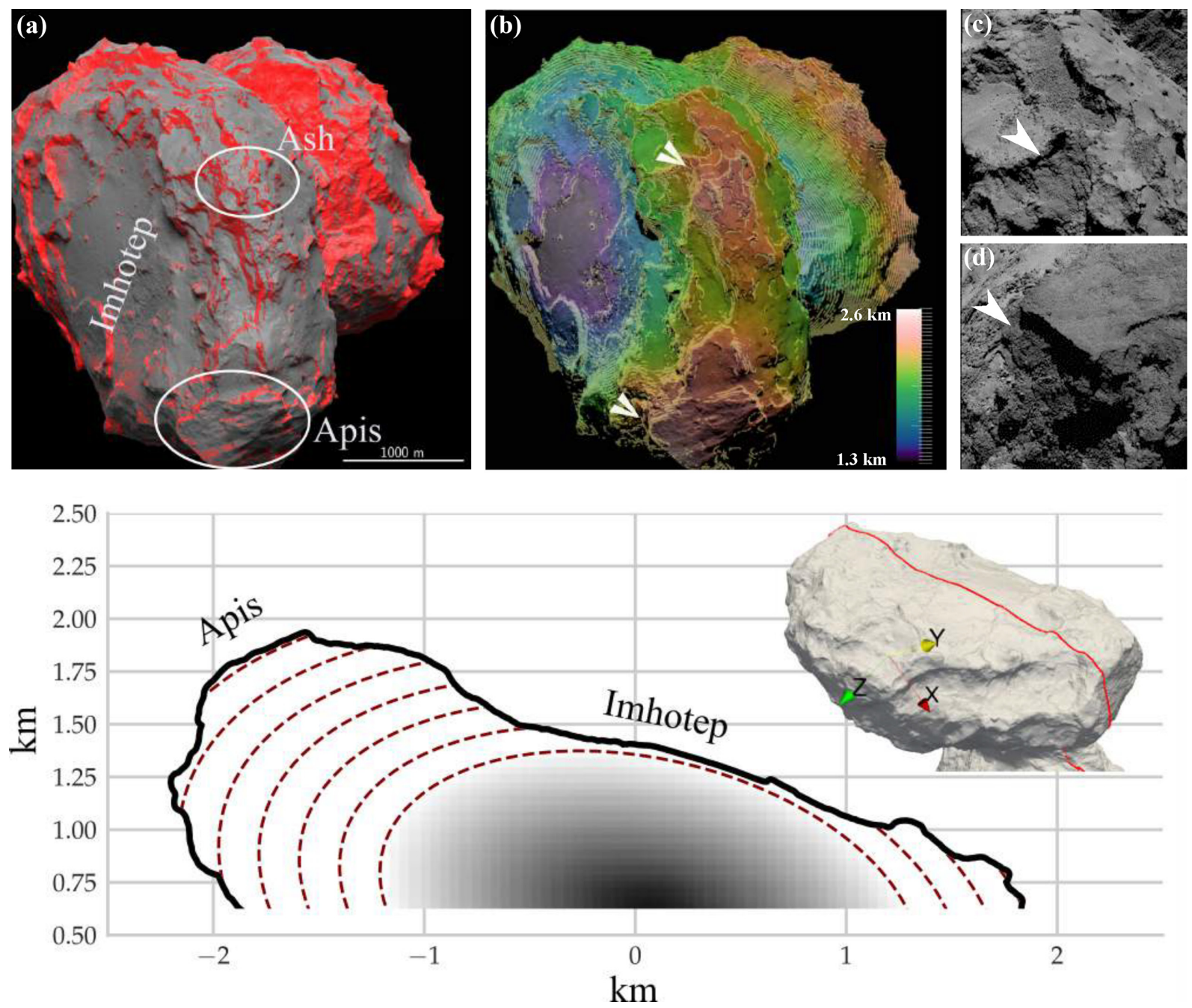

Figure 1. Overview of the innermost and outermost portions of the 67P big lobe: (a) 67P shape model and (b) relative ellipsoid-based model showing innermost and outermost regions of the big lobe (from Penasa et al. 2017, blueish colours correspond to the innermost layers, reddish colours to the outermost ones); (c) close up of Ash region, where top layers appear darker than underlying layers; (d) close up of Apis region, which at the boundary with the underlying Khonsu region is pervaded by goosebump features; (e) structural cross-section of the big lobe (red line on the shape model) that, according to the Elevation Model (EM; Penasa et al. 2017), includes both the higher and the lower layers stacks of the lobe. For interpretation of the colours in this figure, the reader is referred to the web version of this paper.

et al. 2016). The latter groups fine materials with erratic boulders, gravitational accumulation deposits, and remnants of intense sublimation in the form of coarse materials. To support a qualitative comparison between these morphological features and the spectrophotometric results, we produced a detailed geomorphological map for each image set. Mapped units highlight the textural variegation of the surface and are functional to a possible distinction between stacks of layers. Since textural and spectral characteristics of layers' stacks should refer exclusively to autochthonous materials, consolidated outcrops were distinguished by unconsolidated materials, which can have been affected by transfers. Outcropping consolidated materials were further distinguished in relatively unaltered consolidated material, goosebump texture outcrops and degraded outcrops (i.e. highly fractured outcrops, or decametric remnants of sublimation). Coarse deposits include metre-sized boulders mass generated by landslides or intense cometary activity, whereas fine materials define all deposits of the finest fraction of cometary regolith that can occasionally host erratic boulders. Imhotep's roundish features defined by Auger et al. (2015) were mapped separately, the circular rims being possibly autochthonous and the filling fine materials allochthonous. Geomorphological maps of the two images are shown in Figs 2(c)-(d).

NAC images of Table 1 were then used to generate the two multispectral images, renamed dataset $\mathrm{A}$ and dataset $\mathrm{B}$. The OSIRIS standard calibration pipeline described by Tubiana et al. (2015) was used to calibrate images to radiance, expressed in $\mathrm{W} \mathrm{m}^{-2} \mathrm{~nm}^{-1} \mathrm{sr}^{-1}$, then the reflectance (i.e. radiance factor) $\mathrm{I} / \mathrm{F}$ was calculated by means of equation

$\frac{I}{F}=\frac{\pi r_{\mathrm{h}}^{2} I_{\text {meas }}}{F_{\mathrm{s}}}$,

where $I_{\text {meas }}$ is the measured radiance, $F_{\mathrm{s}} / \pi$ is the solar irradiance at $1 \mathrm{au}$ measured at the central wavelength for each filter, and $r_{\mathrm{h}}$ is the heliocentric distance of the comet in astronomical unit. The absolute calibration coefficients of considered filters have a relative uncertainty $\leq 1$ per cent except filter 26 centred at $360 \mathrm{~nm}$, which has a relative uncertainty of 1.7 per cent (Tubiana et al. 2015). 

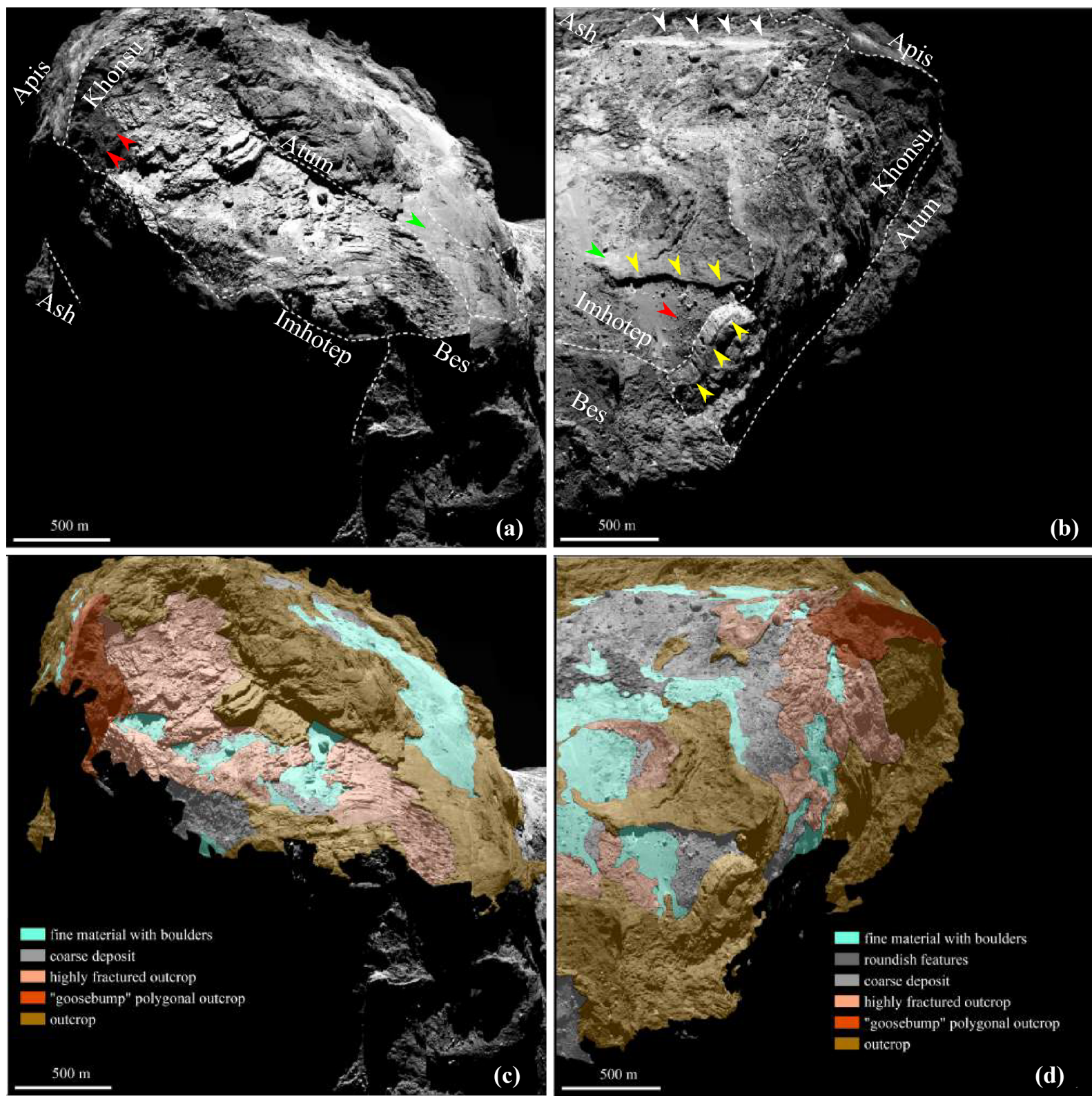

Figure 2. The two OSIRIS NAC images object of our analysis. (a) Image acquired on 2016 Jan 27 at 10:55, labelled NAC_ 2016-01-27T10.53.39.734Z ID30_ 1397549000_ F22: the red arrows indicate the goosebump pattern of Khonsu region. (b) Image acquired on 2016 Jan 27 at 14:11, labelled NAC 2016-01-27T14.09.39.774Z_ ID30_ 1397549000_ F22: the red arrow shows deposits that can be attributed to the erosion of the stand-over outcropping layer, yellow arrows indicate a possible single layer split by an erosional valley, white arrows point to an elongated ledge that cuts the outermost layers' stack. In (c) and (d), the same images are overlaid by the corresponding geomorphological maps. For interpretation of the colours in this figure, the reader is referred to the web version of this paper.

Table 1. OSIRIS NAC sequences of 2016 Jan used in this work. The activity column refers to the OSIRIS science activity. The time column refers to the start time of the first and last observations of the sequence, respectively. Multispectral images achievable with those sequences are renamed dataset A and dataset B. Filter numbers are followed by the corresponding central wavelength in $\mathrm{nm} ; \alpha_{\mathrm{F} 22}$ is the phase angle, $\Delta_{\mathrm{F} 22}$ is the distance between the Rosetta spacecraft and comet $67 \mathrm{P}$ and Scale $\mathrm{F} 22$ is the spatial resolution as from the orange-filter (F22-649.2 nm) image record. Last column indicates the physiographical regions (El-Maarry et al. 2015, 2016) shown by both image sequences.

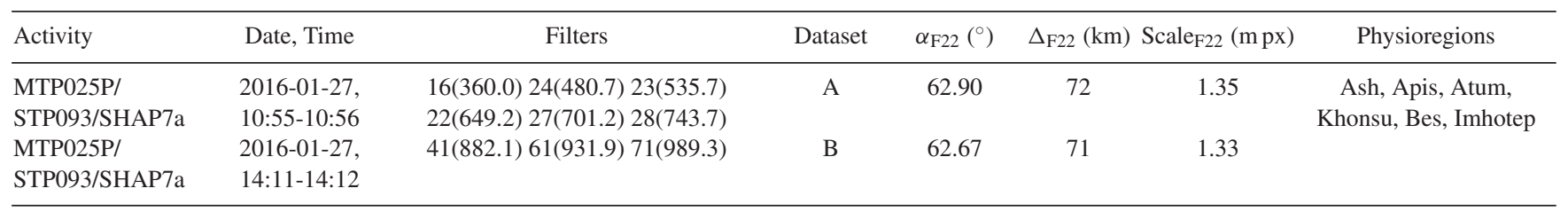


Table 2. RMSE value due to the warping of each filter on the reference filter (i.e. filter 28 for both datasets).

\begin{tabular}{llc}
\hline Dataset & Filters & RMSE \\
\hline A & 16 to 28 & 0.60 \\
22 to 28 & 0.56 \\
23 to 28 & 0.48 \\
24 to 28 & 0.50 \\
27 to 28 & 0.40 \\
41 to 28 & 0.47 \\
61 to 28 & 0.53 \\
& 71 to 28 & 0.47 \\
& 16 to 28 & 0.70 \\
& 22 to 28 & 0.61 \\
& 23 to 28 & 0.53 \\
24 to 28 & 0.50 \\
& 27 to 28 & 0.45 \\
41 to 28 & 0.43 \\
& 61 to 28 & 0.66 \\
& 71 to 28 & 0.51 \\
\hline
\end{tabular}

Images of the same sequence acquired with different filters were co-registered: tie points were collected using ENVI software Imageto-Image function, and images were warped using IDL WARP TRI function (linear interpolation method). 40 tie points per set were identified uniformly along the interested regions. Root mean square errors (RMSE) resulting from warping are listed in Table 2. Each colour sequence was aligned in a multispectral image, obtaining two multispectral images, renamed dataset $\mathrm{A}$ and dataset $\mathrm{B}$, showing different views of the comet. The SPC_ SHAP7_ v1.7 shape model (SPC; Jorda et al. 2016) was used, combined with the most recent NAIF-SPICE kernels and relative IDL code toolkit (Acton 1996; Acton et al. 2018), to produce high-resolution synthetic images and derive the illumination angles. Illumination and topographic effects were corrected using Akimov photometric model (Shkuratov et al. 2011, and reference therein), which has been tested in a previous analysis of the same body (La Forgia et al. 2015). Incidence and emittance angles greater than $80^{\circ}$, corresponding to regions in shadow or very close to the shadow edge, were excluded during the processing. Reflectance values lower than 0.002 , which in shadowed areas result in an abrupt increase of spectral slope, were eventually converted into background values. Corresponding false-colour images are shown in Figs 3(a)-(b).

Multispectral images were firstly used to get spectral slope maps. Spectral slope maps expressed in percent/100 nm were generated between near-infrared (near-IR, $882.1 \mathrm{~nm}$ ) and green $(535 \mathrm{~nm})$ filters, as generally proposed by previous works

$S=\frac{I / F_{\text {NearIR }}-I / F_{\text {Green }}}{\lambda_{\text {NearIR }}-\lambda_{\text {Green }}} \cdot \frac{10000}{I / F_{\text {Green }}}$,

where $I / F_{\text {NearIR }}$ and $I / F_{\text {Green }}$ are the Akimov-corrected radiance factors in the two filters. Spectral slope maps of the two sets are shown in Figs 3(c)-(d).

For the purpose of discerning spectral characteristics of layers stacks, consolidated outcrops constituting layers are here coupled with coarse deposits, which can be easily ascribed to local outcrop sources (e.g. Fig. 2b, red arrow) and should reflect similar spectral behaviours. Fine materials that form deposits, on the other hand, are easily subject to transfer during the cometary activity and, for that reason, can be considered for most allochthonous and useless to define layers spectral characteristics. Therefore, in order to statistically distinguish consolidated materials (including surrounding coarse deposits) from deposits of fine materials, a two-classes maximum likelihood (ML) supervised classification (Richards \& Jia 2006) was performed using all nine available bands. For each class, a group of representative pixels, namely a region of interest (ROI), was suitably defined on multispectral images. Multipolygonal ROIs shown in Figs 4(a)-(b) were selected based on mapped units of Figs 2(c)-(d). The two classes were thus defined by computing mean vector, variance, and covariance of the nine bands of each ROI. Provided a pixel position in the multispectral space, the ML classifier computed the likelihood that the pixel belongs to each class and assigned the pixel to the class of higher probability. The results of the classification of Figs 4(c) and (d) were compared with the geomorphological mapping (Figs $2 \mathrm{c}-\mathrm{d}$ ) and the relative gravitational slope maps (Figs $4 \mathrm{e}-\mathrm{f}$ ); the overall agreement is evident. Indeed, the class of consolidated materials and surrounding coarse deposits (brown class) matches the expected outcropping and coarse units and includes all occurring high-gravitational slope terrains, whereas the class of fine-material deposits (cyan class) corresponds to smooth-surface deposits that for most fill low-gravitational slope terrains. Part of the class attributed to fine materials overlaps smooth areas interpreted as outcrops (Figs $2 \mathrm{a}-\mathrm{b}$, green arrows) and is scattered beyond the identified boundaries, being possibly patches of thin blankets overlaying consolidated material.

Pixels classified as fine materials were then omitted from the two multispectral image, which by doing so became most likely representative of the autochthonous materials (Figs 5a-b). These images were then used to obtain classifications aimed at looking for potential spectral variations related to the comet layering. Specific ROIs were so selected depending on their structural elevation according to the EM (Figs 5c-d). In particular, ROIs that define the outermost stacks of layers were selected at the Khonsu-Apis boundary with a corresponding elevation between 2.3 and $2.5 \mathrm{~km}$ from the structural centre of the lobe, whereas ROIs that define deeper layers were selected on Khonsu and Imhotep regions, at elevations of 2.0-2.1 and 1.9-2.0 km, respectively. The spectral separability between ROIs attributed to different stacks of layers was tested using Jeffries-Matusita and Transformed Divergence separability measurements, which satisfied values higher than 1.9 (Richards \& Jia 2006). ML classification was reiterated, obtaining the classes shown in Figs 6(a)-(b): blue class is representative of the spectral characteristics of the outermost layers, and red class of the spectral characteristics of the innermost layers.

\section{RESULTS}

Geomorphological maps highlighted that Bes, Atum, Apis, and Ash regions are mainly constituted by outcropping consolidated material partly covered by patches of fine materials (Fig. 2), in agreement with the overall geomorphological mapping of Giacomini et al. (2016) and Lee et al. (2016). Imhotep and Khonsu regions appear more variegated, hosting both consolidated material, fragmented outcrops possibly due to intense sublimation, coarse deposits and extensive deposits of fine materials. Between Ash and Imhotep, a $150 \mathrm{~m}$-wide ledge covered by fine materials cuts the stack of layers (Fig. 2b, white arrows). Below the ledge, there are extensive deposits of coarse material and the peculiar group of circular features (Auger et al. 2015) of unclear origin. The large accumulation basin of Imhotep (Auger et al. 2015) is located at the edge of an extended layer (Fig. 2b, yellow arrows) that forms an isolated mesa (Circular Mesa; Hasselmann et al. 2017). In addition, goosebump patterns are present in Khonsu, at the boundary with Imhotep and Apis regions (Fig. 2a, red arrows). According to our photometrically corrected images, outcropping consolidated material forming Imhotep, Bes, 

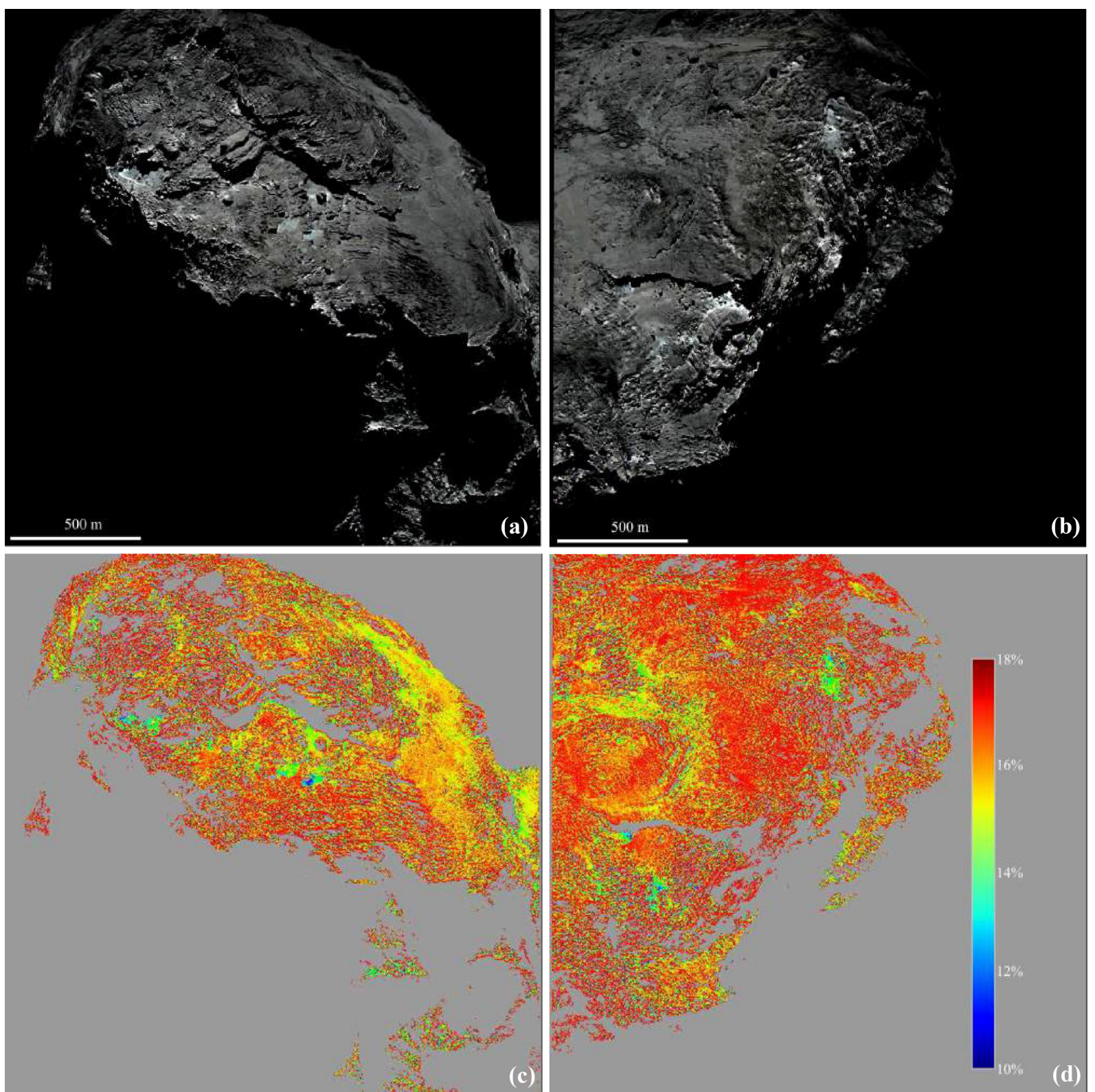

Figure 3. False-colour images of the two sequences obtained from multispectral data (red corresponds to filter 28 centred at $743.7 \mathrm{~nm}$, green corresponds to filter 23 centred at $535.7 \mathrm{~nm}$, blue corresponds to filter 24 centred at $480.7 \mathrm{~nm}$ ). (c)-(d) Spectral slope maps of the two sequences computed with the formula in equation (2): the persistent red (positive) slope along visible and near-infrared wavelengths characteristic of 67P comet (e.g. Fornasier et al. 2015) is confirmed. For interpretation of the colours in this figure, the reader is referred to the web version of this paper.

and Khonsu regions displays a reflectance two times higher than the analogue material constituting Ash, Apis, and Atum regions (e.g. Figs 6c-d).

Post-perihelion data used for this work provide spectral slopes of 17-18 per cent that characterize most of the surface (Fig. 3), with the exception of some fine-material deposits that display a variability from 14 to 18 per cent and restricted areas under 12 per cent where ice patches have been documented (e.g. Barucci et al. 2016; Deshapriya et al. 2016, 2018; Oklay et al. 2016). These results are in good agreement with those by Hasselmann et al. (2017), who used post-perihelion multispectral data with similar phase angle $\left(65^{\circ}\right.$, WAC images of 2016 February 10), and those of Fornasier et al. (2016) who found a nucleus slope of $16.7 \pm 0.4$ averaged over $16 \mathrm{~h}$ of observations on 2016 January 27.
ML classification of coarse and consolidated materials belonging to shells located at different structural elevations of the EM provides two kinds of classes renamed 'outer class' and 'inner class' (respectively, the blue classes and the red classes in Figs 6a-b). The outer class fills Ash, the overhang between Apis and Khonsu, most of Atum region, and isolated large patches in Bes. The inner class is mainly representative of Imhotep, Bes, and Khonsu regions. In terms of classified pixels, the outer class is notably less represented, being one-third of the inner class (Fig. 7). A comparison between the spatial distribution of the classes of Figs 6(a)-(b), the photometrically corrected images (Figs 6c-d) and the geomorphological overview of the area (Figs 2c-d) emphasizes that most of the outer class consists of darker consolidated material, including goosebump textures, whereas the inner class includes highly fractured outcrops, 

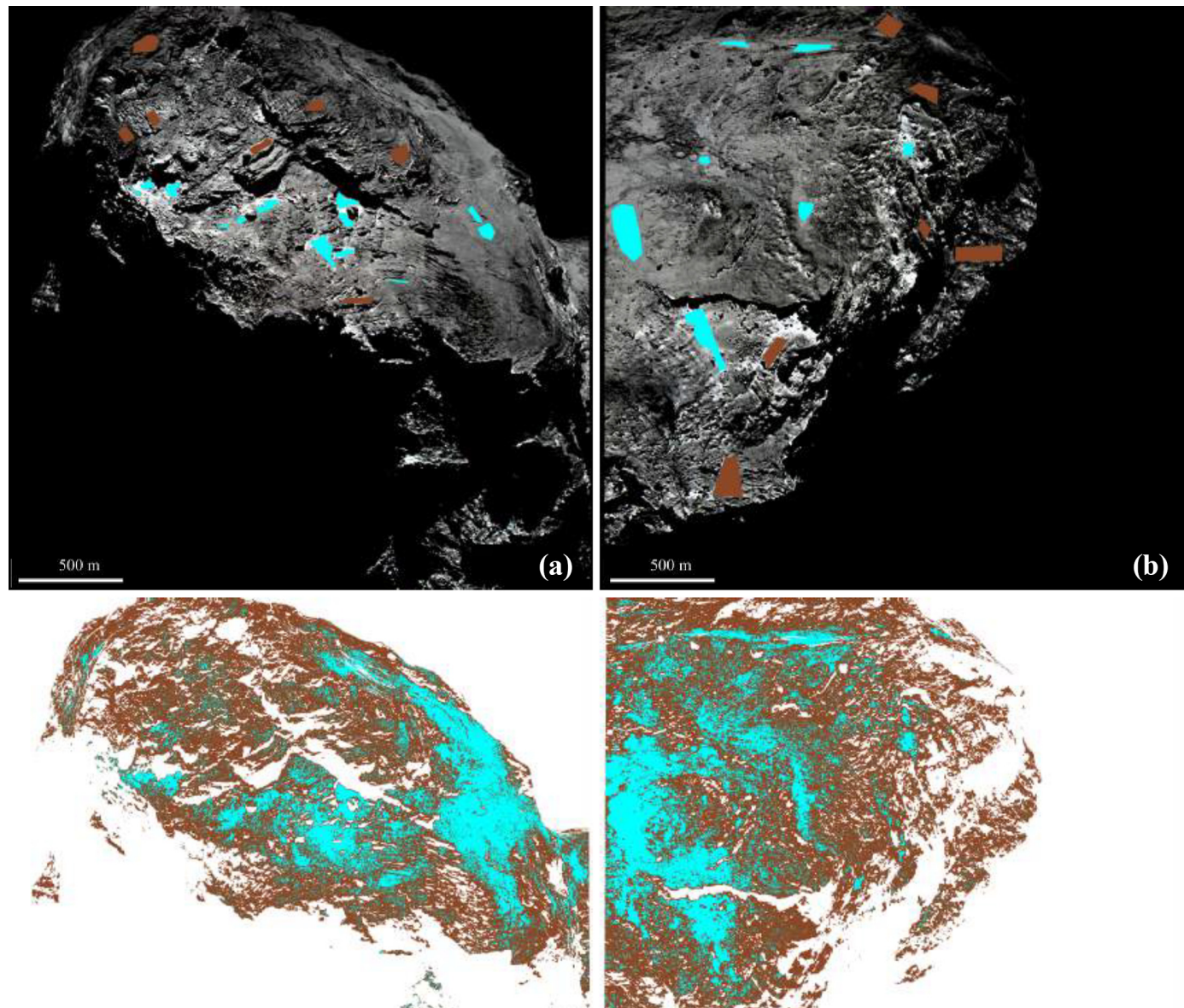

(b)

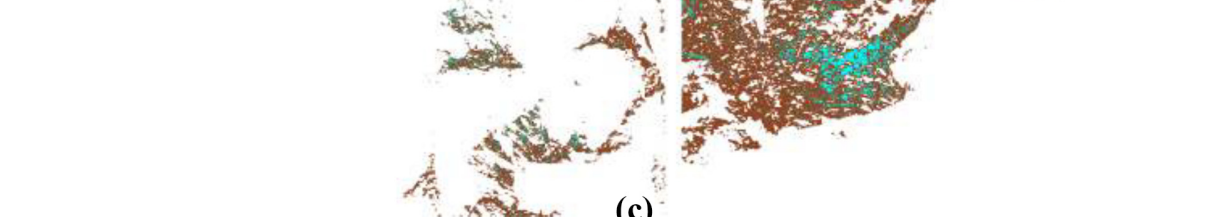

(d)
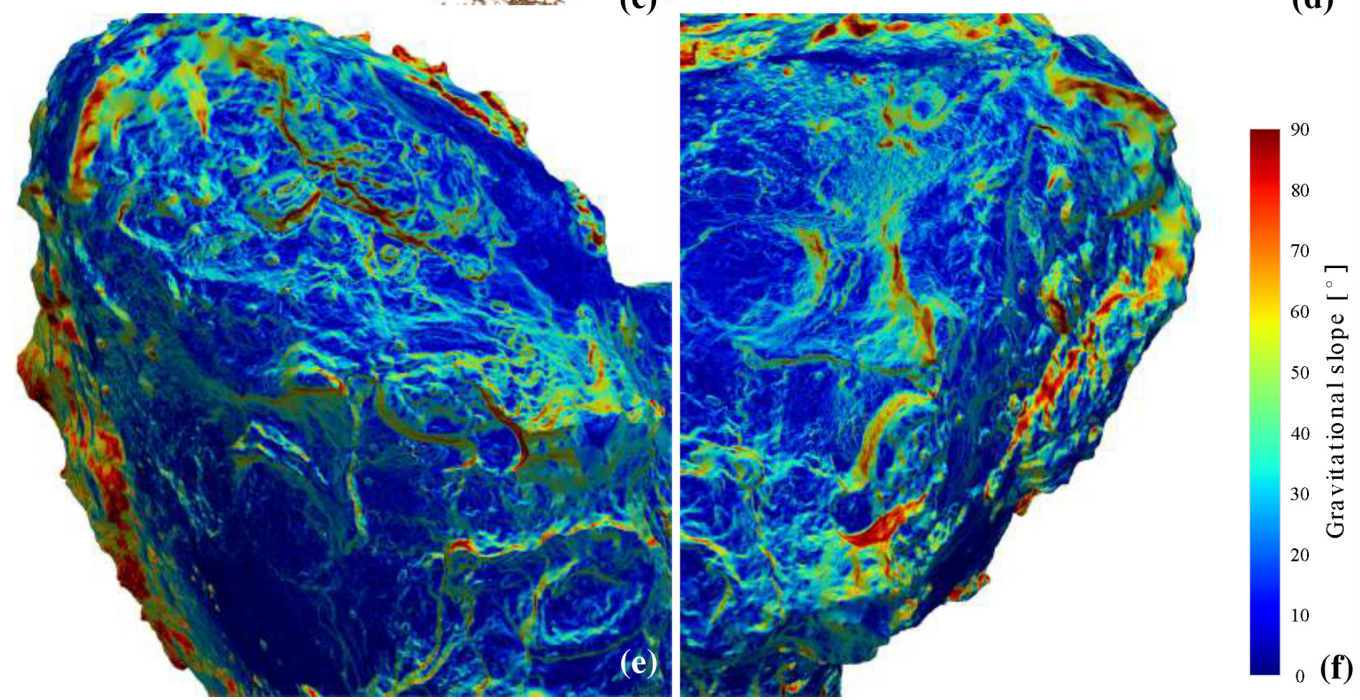

Figure 4. (a)-(b) Photometrically corrected images of filter 22 (centred at $649.2 \mathrm{~nm}$ ) overlapped by multipolygonal ROIs selected based on domains mapped in Fig. 2: cyan ROIs refer to fine materials, brown ROIs refer to consolidated materials. (c)-(d) ML-classified images of fine materials (cyan class) and consolidated materials (brown class). (e)-(f) Gravitational slope maps. For interpretation of the colours in this figure, the reader is referred to the web version of this paper. 

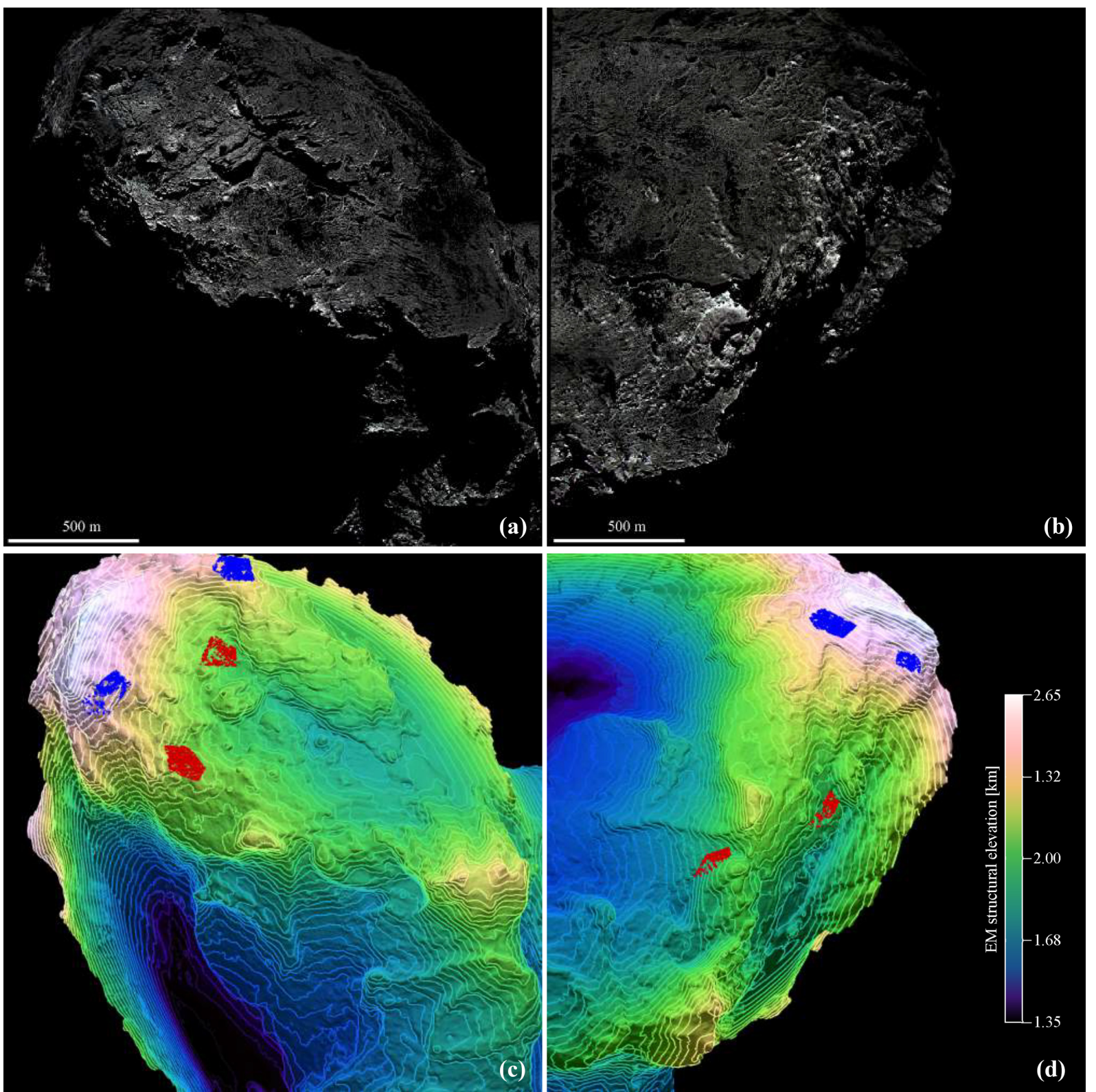

Figure 5. (a)-(b) True-colour images of the two sequences obtained by multispectral data where fine-material deposits have been removed (red corresponds to filter 28 centred at $743.7 \mathrm{~nm}$, green corresponds to filter 23 centred at $535.7 \mathrm{~nm}$, blue corresponds to filter 24 centred at $480.7 \mathrm{~nm}$ ). (c)-(d) The EM overlapped by ROIs selected on the bases of the structural elevation: blue ROIs refer to the outer class, red ROIs refer to the inner class. For interpretation of the colours in this figure, the reader is referred to the web version of this paper.

coarse deposits, and the brighter consolidated material of Bes' cuestas. A mixed/scattered area corresponds to the consolidated Circular Mesa (Hasselmann et al. 2017) at the margin of the accumulation basin of Imhotep (Auger et al. 2015) and to the adjacent coarse deposit (Fig. 6b). By taking into account the pixel distribution in function of the EM structural elevations (Fig. 7), the $2.05-2.10 \mathrm{~km}$ elevation terrains of both classes seem to be particularly exposed, corresponding to the flat surface of a terrace that extends between Imhotep and Khonsu regions. The outer-class pixel distributions show at least a second mode over $2.20 \mathrm{~km}$, at $2.40 \mathrm{~km}$ of elevation (Fig. 7), corresponding to the Apis region. When plotting the percentage of pixels occupied by each class in function of the structural elevation (Fig. 8), the overall prevalence of pixels attributed to the inner class is even clearer. On the other hand, a significant increase of the outer-class pixels to the detriment of the inner-class ones occurs over $2.20 \mathrm{~km}$, reflecting the distribution relative to the second mode.

We found no spatial correlation between inner/outer classes (Figs 6a-b) and either gravitational slopes (Figs 4e-f) or spectral slope distributions (Figs 3c-d). However, checking the absolute average spectra we found that the inner class displays a higher reflectance with respect to the outer one in both multispectral images (Fig. 9, upper panels). Despite the large error bars, the absolute reflectance values seem to be the most significant discriminant factor for the classification. Reflectance values normalized to the green filter (i.e. filter 23 centred at $535.7 \mathrm{~nm}$, Fig. 9, lower panels), indeed, provide both a spectral slope of 18 per cent and do not display significant divergences apart from a small deviation over the near- 


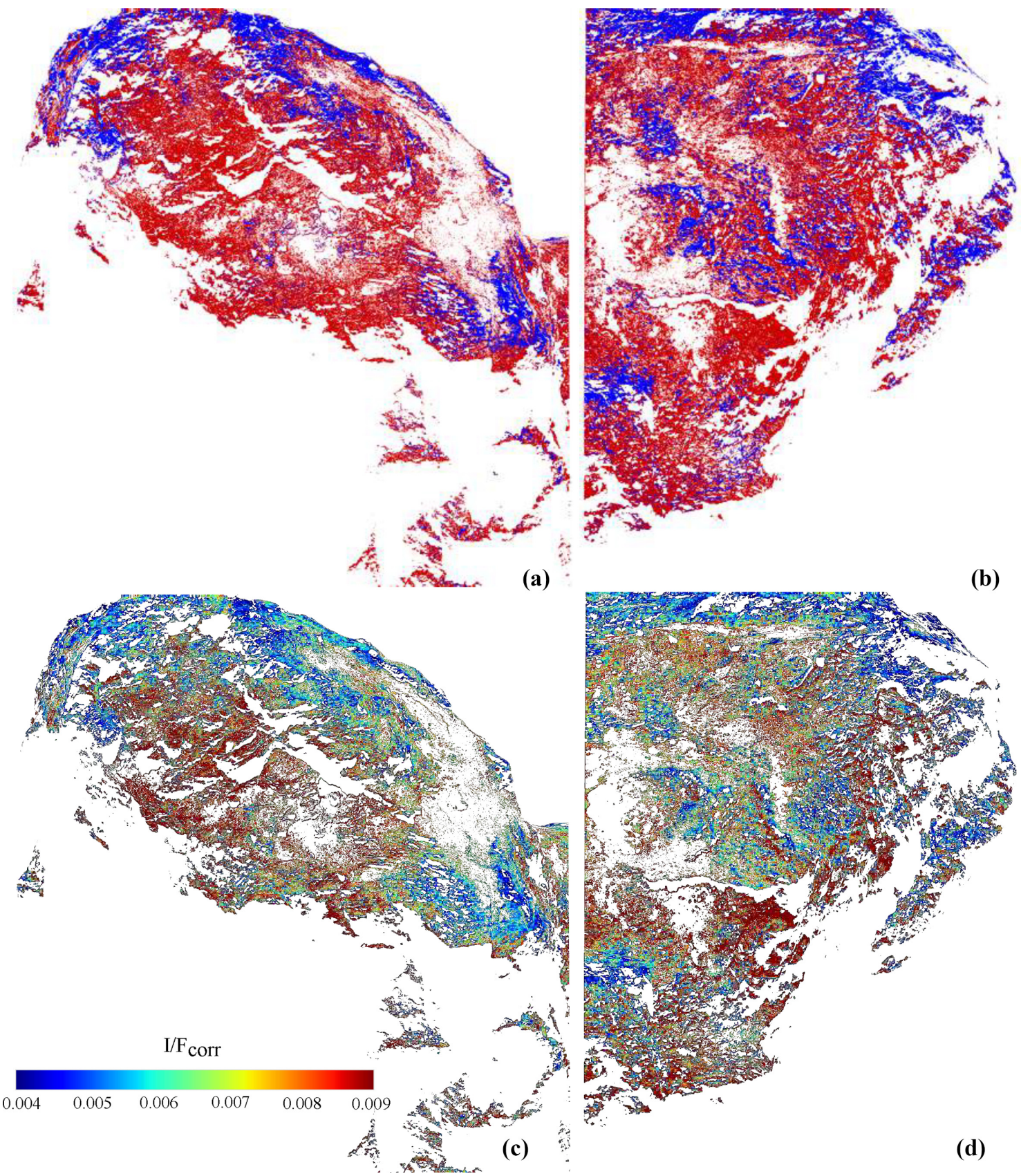

Figure 6. (a)-(b) ML-classified images of coarse and consolidated (autochthonous) materials: blue class is named outer class, red class is named inner class. (c)-(d) Photometric corrected images of filter 22 (centred at $649.2 \mathrm{~nm}$ ) where fine-material deposits have been removed. For interpretation of the colours in this figure, the reader is referred to the web version of this paper.

infrared values. Fine-materials average spectra, which have been integrated for comparison, show even higher absolute reflectance values than those of the outcrop classes. The outer class is spectrally consistent in both images (Fig. 10) because of the proximity of the selected ROIs, whereas both the inner class and fine materials report a relative difference of about 10 per cent in absolute reflectance between the two datasets.

\section{DISCUSSION}

Most of the 67P big-lobe surface detected in our two OSIRIS multispectral images displays a spectral slope of $17-18$ percent along visible and near-infrared wavelengths. The supervised classification of those images allowed to separate outcrops and relative coarse deposits from fine-material deposits, highlighting a lower brightness of layers located at the highest structural level.

In 2014 August, 1 yr before the perihelion passage, when 67P was at 3.6 au and progressive heating and diurnal cycles had a greater role in the activity processes, the surface of irradiated regions like those under study displayed a spectral slope variegation from 13 to 18 per cent (Fornasier et al. 2015) at phase angles of $50^{\circ}$. Approaching the perihelion passage, in late 2015 August, the nucleus became bluer, with a corresponding decrease of about 30 per cent in the mean spectral slope of equatorial regions like Imhotep com- 

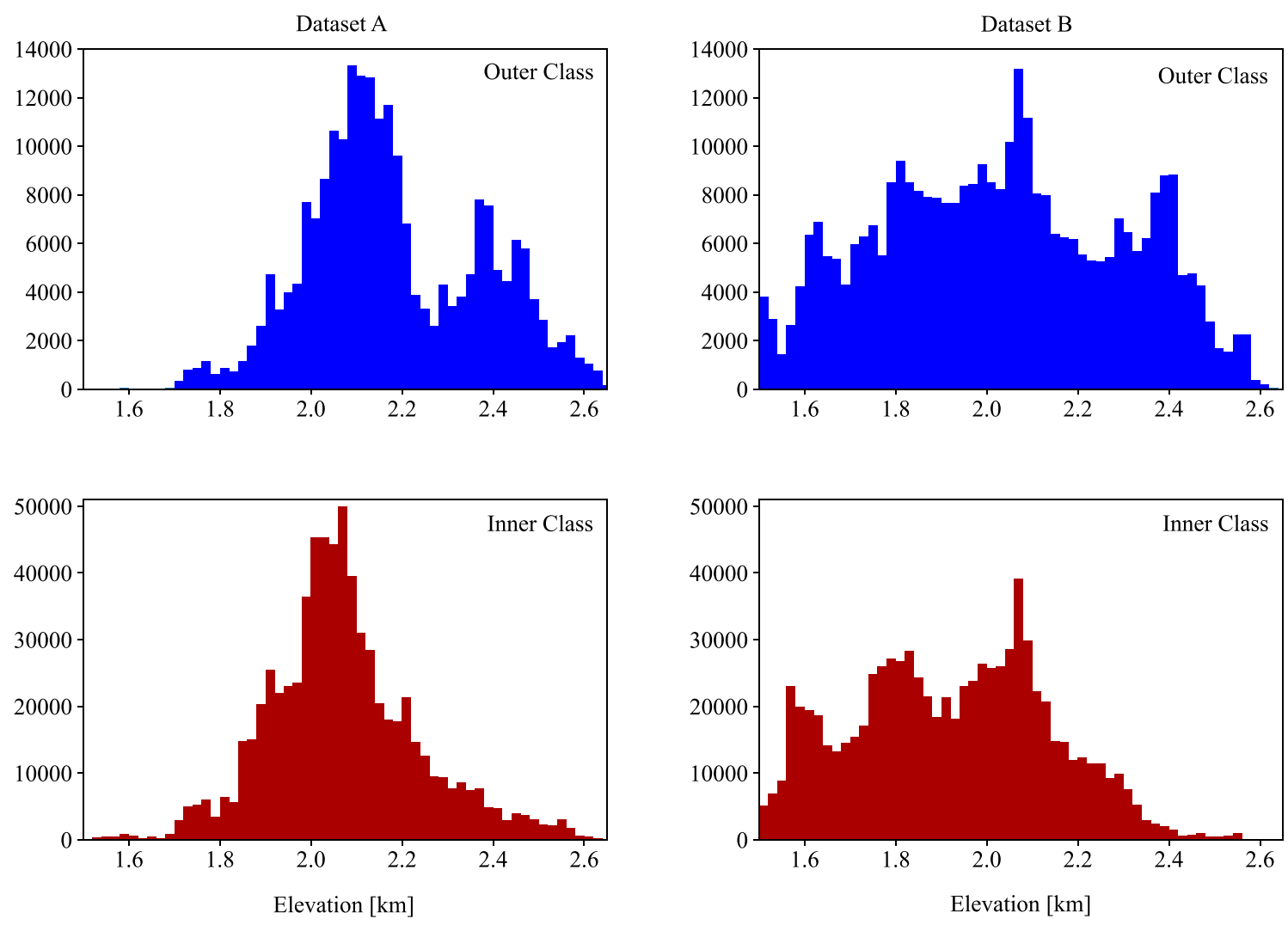

Figure 7. Number of pixels classified per each dataset as a function of the EM structural elevation in $\mathrm{km}$.

pared to 2014 August data (Fornasier et al. 2016), suggesting that the intense activity due to the increased illumination exposed blueslope ice-rich layers. Five months after the perihelion passage, at $2.2 \mathrm{au}$, the surface shown in our image sets results strongly depleted by volatile content, providing higher spectral slopes for most of the terrains, in agreement with results of Fornasier et al. (2016) and Hasselmann et al. (2017). For a rough comparison between pre- and post-perihelion periods, we refer to sequences of 2014 August 5 (Fornasier et al. 2015) and 2015 May 2 (Fornasier et al. 2016) that have phase angles $\left(51^{\circ}\right.$ and $61^{\circ}$, respectively) close to those used in this work (i.e. $\backsim 63^{\circ}$ ). The phase reddening has been proven to be important for the comet and to vary over the orbit (Fornasier et al. 2015, 2016). However, in 2016 January observations, the mean spectral slope values were found to be close to those observed pre-perihelion, at similar phase angles (Fornasier et al. 2016). The volatiles depletion is attested by the reddening of fine-material deposits and outcrops, which varies from 15 to 17 18 per cent (Fig. 3); some circumscribed deposits, on the contrary, maintain their blue spectral attribute (Fig. 3), pointing out long lasting reservoirs of water ice (Oklay et al. 2017). The diffuse reddening reflects a higher surface content of refractories with respect to any pre-perihelion surface study, and prevent any direct comparison of our outputs with pre-perihelion statistical clusters based on spectral slope differences (e.g. Perna et al. 2017). On the other hand, fine materials result steadily brighter than the surrounding consolidated material (Fig. 10), in agreement with pre-perihelion observations of Fornasier et al. (2015).

A higher reflectance of fine materials with respect to the outcrop is also in agreement with pre-perihelion results of La Forgia et al. (2015) on the 67P small lobe, who found a good correlation between reflectance values and all the geomorphological domains, and attributed the spectral variations to compositional variegation and/or texture and grain size effects. In our study, however, the proposed classification highlighted a difference of absolute reflectance between two items (i.e. two stacks of layers) belonging to the same morphological domain (i.e. consolidated material), providing higher values for the innermost of the two (Fig. 9, upper panels). Outcrops, indeed, are evenly distributed between outer and inner classes at different structural elevations in the EM, suggesting that spectral differences are more related to the structural level rather than the degradation stage of the material, which uses to define most of the $67 \mathrm{P}$ geomorphological units. This is particularly evident for the pronounced stack of layers at the boundary among Khonsu, Atum, and Bes regions (Fig. 6). In fact, although equally formed by consolidated material, they display a lower reflectance with respect to the underlying layers, and this is in agreement with the different structural elevation rather than the degradation state. Hence, the different brightness of the consolidated material can be associated to a different composition or a different texture of the same morphological domain, where texture uses to refer to the surface roughness and the porosity of consolidated materials.

On the other hand, the Ash region and its boundary with Imhotep, the outcrop of which is included in the outer class, underwent a brightness decrease coinciding with the perihelion passage; the decrease has been attributed to a preferential deposition of volatiledepleted dust on certain topological features (Hasselmann et al. 2017). This interpretation is supported by the fact that the mentioned area is located on the northern hemisphere, which during the perihelion passage was inactive and thus able to accumulate airfalls that originated from the active southern regions (Keller et al. 

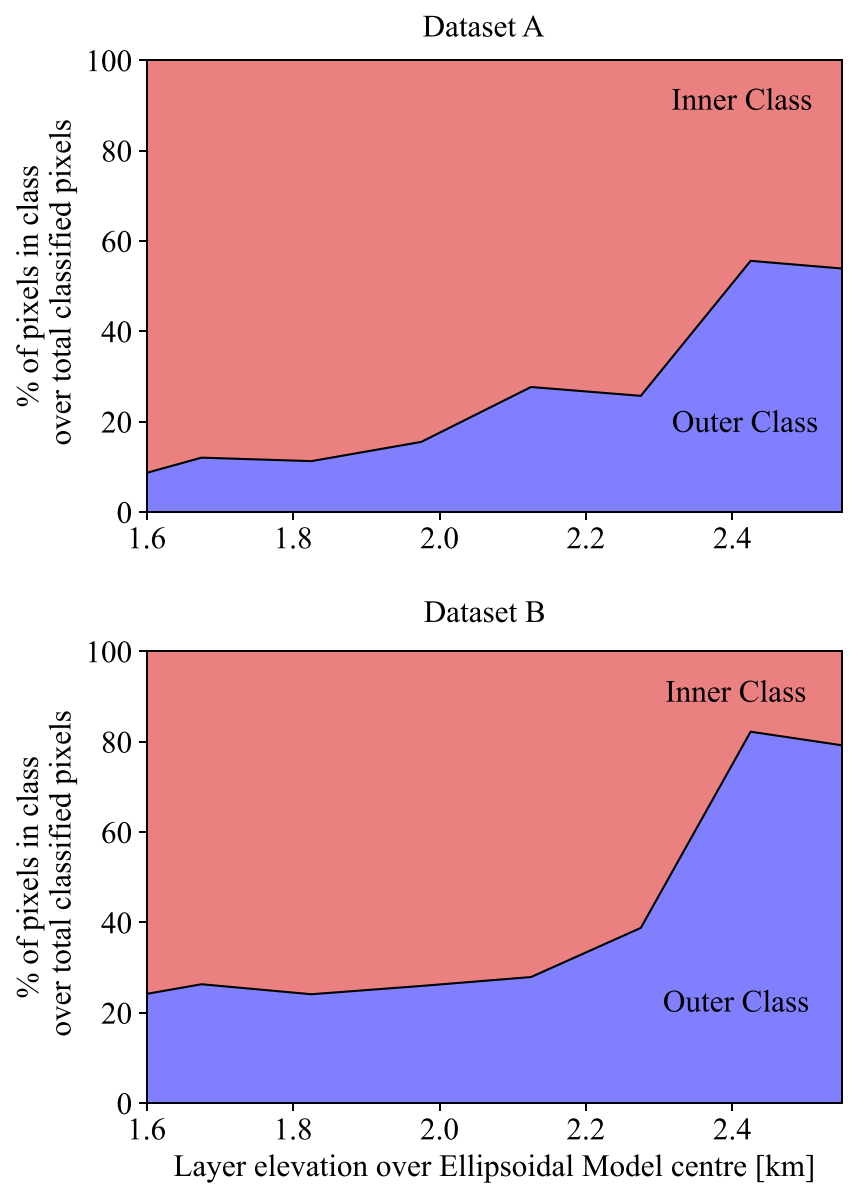

Figure 8. Percentage of pixels in each class over total classified pixel in function of the EM structural elevation.

2017). However, the concurrent darkening of outer-class southern areas like those at the boundary among Bes, Khonsu, and Imhotep regions (Figs 6a and c) cannot be due to such a seasonal airfall, being southern regions active during the perihelion passage. Again, the different brightness of layers stacks could reflect a different composition or textural pattern of the consolidated materials.

Ruling out whether these are the results of an evolutionary process or are instead intrinsic and primordial characteristics of the cometary material is a challenging task. Indeed, any variation that concerns the nucleus composition or texture could have occurred (i) during the nucleus accretion and/or before the volume loss of the outer layers pointed out by Penasa et al. (2017), implying that the inner layers reveal their intrinsic brighter nature coinciding with those volume removals (Fig. 11a), or (ii) during a process selectively involving parts of an homogeneous cometary nucleus (Fig. 11b).

A different composition of the consolidated material could be related to a relatively higher content of refractories (Capaccioni et al. 2015) in the outer layers, making those layers less bright. This implies that during its accretion, this $67 \mathrm{P}$ lobe could have encountered regions of the protoplanetary Solar Nebula with different abundances of refractories, or that the same Solar-system reservoir could have experienced a progressive depletion of volatiles (Fig. 11a). Alternatively, the depletion of volatiles could have occurred during the perihelion passages, and the surface of inner layers would become less depleted because it could have been exposed later, through the erosion of the same outer layers (Fig. 11b). Both hypotheses seem to be supported by the fact that all of the ice patches documented in this area by other authors (e.g. Deshapriya et al. 2016, 2018; Oklay et al. 2016) correspond to the inner stacks (Figs 3 and 6), which turns out to remain rich in volatiles.

A correlation between brightness and texture is likewise conceivable. In fact, consolidated materials with relatively higher surface roughness can provide lower reflectance, whereas consolidated material with higher porosity could have higher brightness (Harloff \& Arnold 2001). Assuming a similar composition and grain-arrangement of these material (e.g. mixtures of ice and refractory materials rather than agglomerates of ice grains with refractory cores; Kossacki et al. 2015), whenever the roughness is greater than the optical wavelength of observation, the brightness is controlled by the effects of interparticle multiple scattering, decreasing with increased roughness (Ogilvy et al. 1987). Both sublimation and thermally induced fracturing can likely affect the surface roughness of a comet, according to the local topography that governs the insolation. On the other hand, given similarly oriented surfaces with respect to the solar illumination, a different roughness of stacked layers could have been likely produced by a different degree of thermally induced fracturing. Particularly, the outer layers could have undergone a more extensive fracturing due to their different rheological properties or else longer thermal exposure. The former case would suggest that outer layers are intrinsically more brittle (Fig. 11a), whereas the latter would suggest that inner layers were exposed later to the thermal activity (Fig. 11b).

Similarly, all other factors being equal, a lower porosity could be invoked for the darker outer layers. This hypothesis is consistent with the hypothesis that the nucleus of 67P is heterogeneous (e.g. Vincent et al. 2015; Gutiérrez et al. 2016; Jorda et al. 2016) and in particular that, on each pre-junction lobe, less porous concentric layers could have formed around a more porous interior (Davidsson et al. 2016; Wahlberg Jansson \& Johansen 2017). An increase of porosity with depth is also one of the conclusions of Ciarletti et al. (2015) concerning the small lobe, where the Rosetta CONSERT (Comet Nucleus Sounding Experiment by Radiowave Transmission; Kofman et al. 2015) experiment took place. Assuming that the two lobes formed in a similar manner and experienced similar processes, a higher porosity of the inner layers could be expected also for the big lobe (Fig. 11a). Conversely, a variation of porosity induced subsequently to the comet formation (Fig. 11b) and implying a higher porosity for the inner layers has not been proposed yet.

On the other hand, approximating the highly porous cometary surface material (e.g. 81-84 per cent on Ash and Imhotep regions; Feller et al. 2016; Hasselmann et al. 2017) to a poorly welded material with few intimate contacts between the constituents, one could argue that even a different grain size of the aggregate can produce a different surface reflectance (Hapke 1993). Although a different size distribution of structural elevations has not been modelled in recent formation models (e.g. Blum et al. 2017), concerning our observations, the darker outer layers would correspond to a relatively larger grain size of the cometary material with respect to the brighter inner layers. The variation could pertain to the particles that form the aggregates or to the pebbles that form the cometary nucleus, whether it occurred during the lobe accretion (Fig. 11a) or during the perihelion passages (Fig. 11b). The lower brightness of the outer layers could correspond to a relative enrichment of refractories, being usually larger than ice particles (e.g. Kataoka et al. 2013).

In any case, the texture of the outcrop seems to be a substantial factor for the reflectance dichotomy observed in this study (Fig. 6), whether it is intrinsic or induced. Our overall picture describes the 

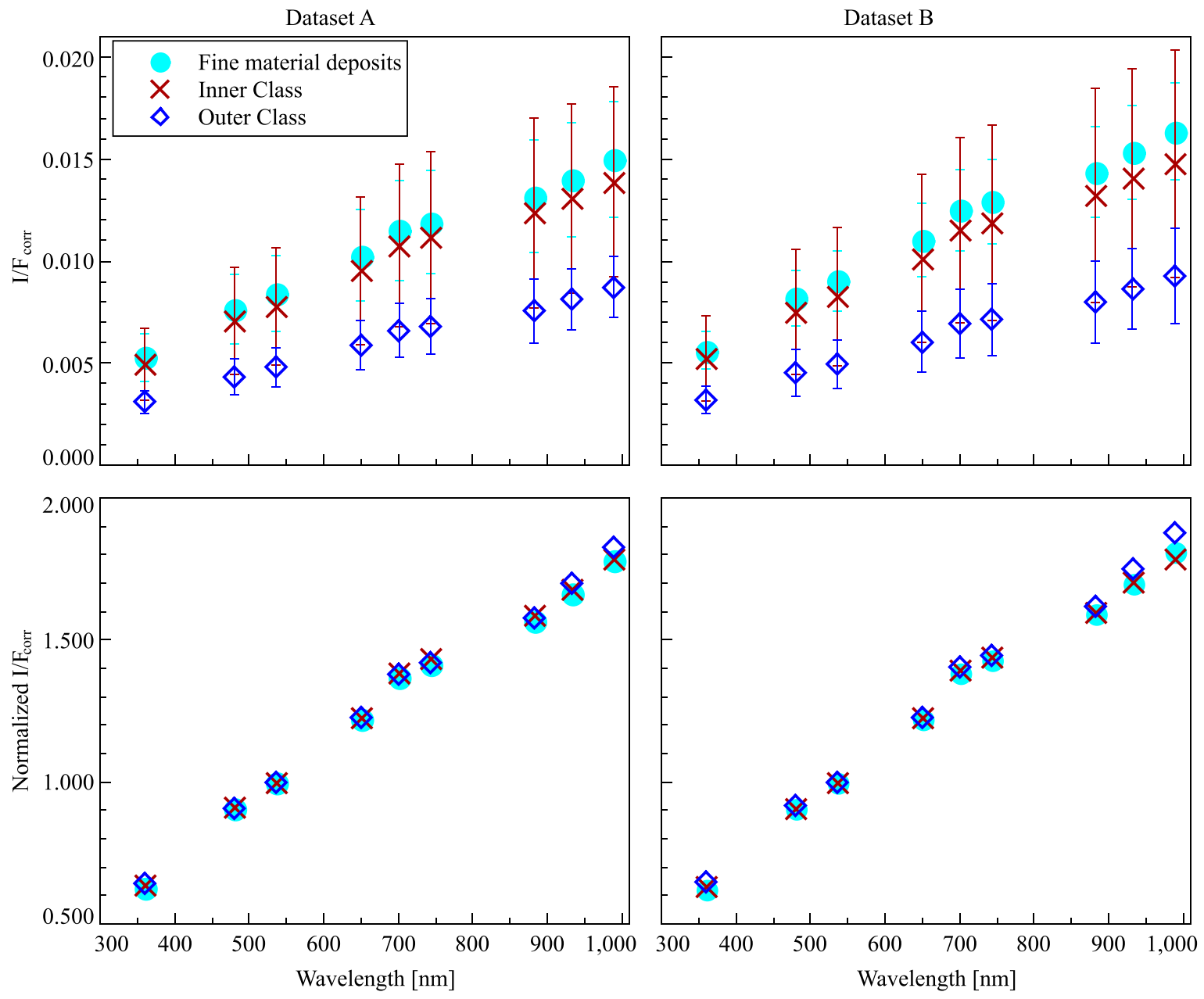

Figure 9. Absolute (top panel) and normalized (bottom panels) average spectra of fine materials, inner, and outer classes. The increase of flux around $700 \mathrm{~nm}$ that characterizes all the spectra of Fig. 7 was already described by Fornasier et al. (2015) as linked to cometary activity and attributed to $\mathrm{H}_{2} \mathrm{O}^{+}$and/or $\mathrm{NH}_{2}$ emission in the very inner coma.



Figure 10. Comparison of absolute average spectra of fine materials, inner, and outer classes of the two sequences: solid lines refer to spectra of dataset A, dashed lines refer to spectra of dataset B.
67P big lobe as formed by stacks of layers that display different brightness. We attribute the brightness differences to the composition or the texture variegations of the consolidated materials, supporting the idea that a localized removal of large volumes of the outer layers uncovered an innermost structure (Penasa et al. 2017) that underwent common surface processes at a later stage.

\section{CONCLUSIONS}

The Rosetta mission has revealed for comet 67P a layered nucleus with an overall dark featureless surface. Two sets of post-perihelion OSIRIS-NAC images having similar phase angle and distance of acquisition were used to obtain nine-filters multispectral images of the 67P comet big lobe. Multispectral data were used to create spectral slope maps and to apply an ML supervised classification of the area in order to study a possible variegation as function of the layers elevation provided by the EM proposed by Penasa et al. (2017). Geomorphological sketches were provided to distinguish autochthonous and allochthonous materials. Two classes of autochthonous terrains were proposed, distinguished by spectral properties, spatial distributions, and structural levels; the outer class was confirmed to be darker and mainly localized on the outermost 
Primordial variegation

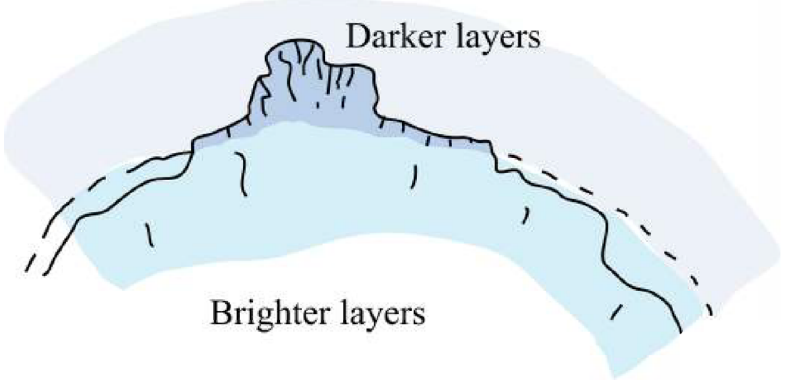

Evolutionary variegation

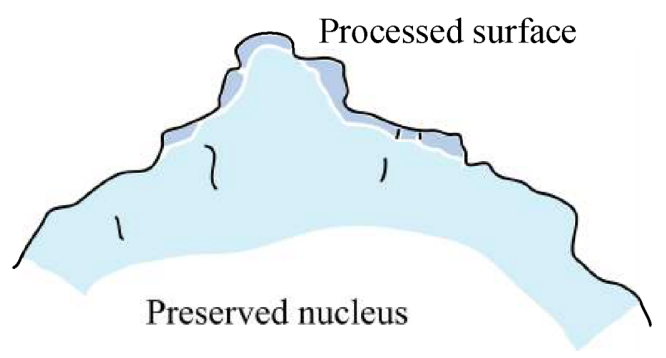

Figure 11. Two possible scenarios on the occurrence of the brightness difference between inner and outer layers of 67P big lobe. On the left, the variegation occurred during the nucleus accretion and before the volume loss of the outer layers, implying that the preserved inner layers were revealed because of later exhumations; on the right, the variegation occurred selectively on a homogeneous cometary surface.

stack of layers of the big lobe, distributed along Ash, Apis, and the tops of Khonsu, Atum, and Bes regions; the inner class was confirmed to be brighter at all wavelengths and diffused on the innermost outcrops of Imhotep, Bes, and Khonsu. The photometric variegation observed between the two stacks is then essentially associated with the different surface brightness. This variegation is most likely ascribable to a different texture of the consolidated material forming the nucleus or to a different content of refractories, rather than to a selective deposition of bright airfall on the outcrop. The brighter innermost structure is currently revealed due to the removal of large volumes of the outer layers (Penasa et al. 2017).

Further analyses of 67P multispectral data that includes both lobes could clear if those different properties of the cometary material were emplaced during the accretion or are induced by later processes.

\section{ACKNOWLEDGEMENTS}

We are grateful to Paul Helfenstein for corrections that improved our paper. OSIRIS was built by a consortium of the Max-Planck-Institut für Sonnensystemforschung, in Göottingen, Germany, CISASUniversity of Padova, Italy, the Laboratoire d'Astrophysique de Marseille, France, the Instituto de Astrofisica de Andalucia, CSIC, Granada, Spain, the Research and Scientific Support Department of the European Space Agency, Noordwijk, The Netherlands, the Instituto Nacional de Tecnica Aeroespacial, Madrid, Spain, the Universidad Politechnica de Madrid, Spain, the Department of Physics and Astronomy of Uppsala University, Sweden, and the Institut für Datentechnik und Kommunikationsnetze der Technischen Universität Braunschweig, Germany. The support of the national funding agencies of Germany (DLR), Italy (ASI), France (CNES), Spain (MEC), Sweden (SNSB), and the ESA Technical Directorate is gratefully acknowledged. We thank the ESA teams at ESAC, ESOC, and ESTEC for their work in support of the Rosetta mission.

\section{REFERENCES}

Acton C. H., 1996, Planet. Space Sci., 44, 65

Acton C., Bachman N., Semenov B., Wright E., 2018, Planet. Space Sci., 150,9

Auger A.-T. et al., 2015, A\&A, 583, A35

Barucci M. A. et al., 2016, A\&A, 585, A102

Blum J. et al., 2017, MNRAS, 469, S755

Capaccioni F. et al., 2015, Science, 347, aaa0628
Ciarletti V., Levasseur-Regourd A. C., Lasue J., Statz C., Plettemeier D., Hérique A., Rogez Y., Kofman W., 2015, A\&A, 583, A40

Coradini A. et al., 2007, Space Sci. Rev., 128, 529

Davidsson B. J. R. et al., 2016, A\&A, 592, A63

De Sanctis M. C. et al., 2015, Nature, 525, 500

Deshapriya J. D. P. et al., 2016, MNRAS, 462, S274

Deshapriya J. D. P. et al., 2018, A\&A, 613, A36

El-Maarry M. R. et al., 2015, A\&A, 583, A26

El-Maarry M. R. et al., 2016, A\&A, 593, A110

Feller C. et al., 2016, MNRAS, 462, S287

Fornasier S. et al., 2015, A\&A, 583, A30

Fornasier S. et al., 2016, Science, 2671, aag2671

Fornasier S. et al., 2017, MNRAS, 469, S93

Giacomini L. et al., 2016, MNRAS, 462, S352

Gutiérrez P. J., et al., 2016, A\&A, 590, A46

Hapke B., 1993, Theory of Reflectance and Emittance Spectroscopy. Cambridge Univ. Press, , Cambridge

Harloff J., Arnold G., 2001, Planet. Space Sci., 49, 191

Hasselmann P. H. et al., 2017, MNRAS, 469, S550

Jorda L. et al., 2016, Icarus, 277, 257

Jutzi M., Benz W., 2017, A\&A, 597, A62

Kataoka A., Tanaka H., Okuzumi S., Wada K., 2013, A\&A, 554, A4

Keller H. U. et al., 2007, Space Sci. Rev., 128, 433

Keller H. U. et al., 2017, MNRAS, 469, S357

Kofman W. et al., 2015, Science, 349, aab0639

Kossacki K. J., Spohn T., Hagermann A., Kaufmann E., Kührt E., 2015, Icarus, 260, 464

La Forgia F. et al., 2015, A\&A, 583, A41

Lai I. L. et al., 2016, MNRAS, 462, S533

Lee J. C. et al., 2016, MNRAS, 462, S573

Lucchetti A. et al., 2016, A\&A, 585, L1

Lucchetti A. et al., 2017, MNRAS, 469, S238

Massironi M. et al., 2015, Nature, 526, 402

Ogilvy J. A., 1987, Rep. Prog. Phys., 50, 1553

Oklay N. et al., 2015, A\&A, 586, A80

Oklay N. et al., 2016, 462, S394

Oklay N. et al., 2017, MNRAS, 469, S582

Pajola M. et al., 2016, A\&A, 592, L2

Pajola M. et al., 2017a, Nat. Astron., 1, 92

Pajola M. et al., 2017b, MNRAS, 469, S636

Penasa L. et al., 2017, MNRAS, 469, S741

Perna D. et al., 2017, A\&A, 600, A115

Richards J. A., Jia X., 2006, Remote Sensing Digital Image Analysis: An Introduction. Springer-Verlag, Berlin

Rickman H. et al., 2015, A\&A, 583, A44

Shkuratov Y., Kaydash V., Korokhin V., Velikodsky Y., Opanasenko N., Videen G., 2011, Planet. Space Sci., 59, 1326

Sierks H. et al., 2015, Science, 347, aaa1044 
Thomas N. et al., 2015, Science, 347, aaa0440

Tubiana C. et al., 2015, A\&A, 583, A46

Tubiana C., Böhnhardt H., Agarwal J., Drahus M., Barrera L., Ortiz J. L., 2011, A\&A, 527, A113

Vincent J. B. et al., 2015, Nature, 523, 63

Vincent J. B. et al., 2016, MNRAS, 462, S184

Wahlberg Jansson K., Johansen A., 2017, MNRAS, 469, S149

${ }^{1}$ Center of Studies and Activities for Space (CISAS) 'G. Colombo', University of Padova, Via Venezia 15, I-35131 Padova, Italy

${ }^{2}$ Department of Physics and Astronomy 'G. Galilei', University of Padova, Vic. Osservatorio 3, I-35122 Padova, Italy

${ }^{3}$ Geosciences Department, University of Padova, via G. Gradenigo 6, I35131 Padova, Italy

${ }^{4}$ Department of Physics and Astronomy 'G. Galilei', University of Padova, via Marzolo 8, I-35131 Padova, Italy

${ }^{5}$ CNR-IFN UOS Padova LUXOR, Via Trasea 7, I-35131 Padova, Italy

${ }^{6}$ LESIA, Observatoire de Paris, PSL Research University, CNRS, Univ. Paris Diderot, Sorbonne Paris Cité, UPMC Univ. Paris 06, Sorbonne Universités, 5 place Jules Janssen, F-92195 Meudon, France

${ }^{7}$ INAF-OAPD Astronomical Observatory of Padova, Vic. Osservatorio 5, I-35122 Padova, Italy

${ }^{8}$ Deutsches Zentrum für Luft- und Raumfahrt (DLR), Institut für Planetenforschung, Rutherfordstraße 23, D-12489 Berlin, Germany

${ }^{9}$ Max Planck Institute for Solar System Research, Justus-von-Liebig-Weg 3, D-37077 Göttingen, Germany

${ }^{10}$ Laboratoire d'Astrophysique de Marseille, UMR 7326, CNRS \& Aix Marseille Université, F-13388 Marseille Cedex 13, France

${ }^{11}$ Centro de Astrobiologia, CSIC-INTA, E-28850 Torrejon de Ardoz, Madrid, Spain

${ }^{12}$ International Space Science Institute, Hallerstrasse 6, CH-3012 Bern, Switzerland

${ }^{13}$ Scientific Support Office, European Space Research and Technology Centre/ESA, Keplerlaan 1, Postbus 299, NL-2201 AZ Noordwijk ZH, the Netherlands
${ }^{14}$ Jet Propulsion Laboratory, M/S 183-301, 4800 Oak Grove Drive, Pasadena, CA 91109, USA

${ }^{15}$ LATMOS, CNRS/UVSQ/IPSL, 11 Boulevard d'Alembert, F-78280 Guyancourt, France

${ }^{16}$ Department of Astronomy, University of Maryland, College Park, MD 20742-2421, USA

${ }^{17}$ Department of Industrial Engineering, University of Padova, Via Venezia 1, I-35131 Padova, Italy

${ }^{18}$ Faculty of Engineering, University of Trento, via Mesiano 77, I-38121 Trento, Italy

${ }^{19}$ INAF-Osservatorio Astronomico di Trieste, via Tiepolo 11, I-34143 Trieste, Italy

${ }^{20}$ Aix Marseille Université, CNRS, LAM (Laboratoire d'Astrophysique de Marseille) UMR 7326, F-13388 Marseille, France

${ }^{21}$ Instituto de Astrofísica de Andalucía (CSIC), c/ Glorieta de la Astronomia s/n, E-18008 Granada, Spain

${ }^{22}$ Graduate Institute of Astronomy, National Central University, 300 ChungDa Rd, Chung-Li 32054, Taiwan

${ }^{23}$ Space Science Institute, Macau University of Science and Technology, Avenida Wai Long, Taipa, Macau

${ }^{24}$ Institut für Geophysik und extraterrestrische Physik, Technische Universität Braunschweig, Mendelssohnstr. 3, D-38106 Braunschweig, Germany ${ }^{25}$ Operations Department, European Space Astronomy Centre/ESA, PO Box 78, E-28691 Villanueva de la Cañada (Madrid), Spain

${ }^{26}$ Physikalisches Institut der Universität Bern, Sidlerstr. 5, CH-3012 Bern, Switzerland

${ }^{27}$ Center for Space and Habitability, University of Bern, CH-3012 Bern, Switzerland

This paper has been typeset from a $\mathrm{TE}_{\mathrm{E}} \mathrm{X} / \mathrm{AT} \mathrm{E} \mathrm{X}$ file prepared by the author. 